\title{
El Papel de la Educación Financiera en la Formación de Profesionales de la Ingeniería
}

\author{
Fernando Villada, Jesús M. López-Lezama y Nicolás Muñoz-Galeano \\ Universidad de Antioquia, Facultad de Ingeniería, Departamento de Ingeniería Eléctrica, Calle 67 No. 53 - \\ 108, Oficina 20-405, Medellín, Colombia (e-mail: fernando.villada@udea.edu.co; \\ jmaria.lopez@udea.edu.co; nicolas.munoz@udea.edu.co)
}

Recibido Sep. 5, 2016; Aceptado Nov. 14, 2016; Versión final Dic. 15, 2016, Publicado Abr. 2017

\begin{abstract}
Resumen
En este artículo se analiza la importancia de incluir la educación financiera en el proceso de formación de los profesionales de la ingeniería. La educación financiera se hace cada vez más relevante dado el número creciente de productos que ofrecen los mercados financieros y su efecto en el desarrollo económico de los estados y la calidad de vida de sus ciudadanos. Es evidente que una de las principales causas de las últimas crisis económicas mundiales ha sido la carencia de este tipo de educación. Se analiza el concepto de educación financiera, su relevancia en el mundo moderno y su relación con la ingeniería económica. Finalmente, se describen los principales aspectos que deben ser incluidos en los currículos de ingeniería y se recomiendan algunas medidas para la puesta en práctica de estos conceptos.
\end{abstract}

Palabras clave: educación financiera; formación de ingenieros; ingeniería económica; proyecto de aula

\section{The Role of Financial Education in the Training of Engineering Professionals}

\begin{abstract}
This paper discusses the importance of including financial education in the training process of engineering professionals. Financial education is becoming more relevant every day given the growing number of products offered by the financial markets and its effect on the economic development of the states and the quality of life of its citizens. It is evident that one of the main causes of the recent economic crisis has been the lack of this type of education. The concept of financial education, its relevance in the modern world and its relationship with the engineering economy are analyzed. Finally, the main aspects that should be included in the curricula of engineering are described and certain measures for the implementation of these concepts are recommended.
\end{abstract}

Keywords: financial education; engineering training; engineering economy; classroom project 


\section{INTRODUCCIÓN}

La sociedad capitalista obliga a los individuos a tomar continuamente decisiones financieras, las cuales en mayor a menor grado pueden afectar el futuro de cada persona. Desde niños tenemos que distribuir adecuadamente nuestra mesada, al comienzo de nuestra vida laboral debemos saber administrar nuestro salario, empezar a tomar decisiones de ahorro e inversión, lo mismo que de endeudamiento. Todo esto requiere de una adecuada educación financiera porque cualquiera de estas decisiones puede impactar considerablemente, de forma positiva o negativa, el logro de las metas personales.

La educación financiera ha venido tomando mayor relevancia en las últimas décadas. Diferentes factores sociales, económicos y demográficos han impulsado esta tendencia. Dentro de estos se pueden mencionar: i) Endeudamiento creciente por parte de individuos debido a mayor oferta de créditos, ii) Aumento en oferta y complejidad de elementos financieros iii) Aumento de la inestabilidad laboral, iv) Aumento de la esperanza de vida, y v) Reducción de coberturas públicas de pensiones. En este contexto, una adecuada educación financiera es imperativa para mejorar el estándar de vida de los individuos. Según algunos expertos, fue precisamente la carencia de la misma la principal causa de la última crisis económica en Estados Unidos y Europa, originada en el mercado inmobiliario (Melvin y Taylor, 2009; Dwyer y Lothian, 2012; Bartram y Bodnar, 2009). No se pueden desconocer los abusos del sistema financiero, pero fueron finalmente las personas naturales quienes tomaron la decisión de solicitar deudas por encima de sus posibilidades, adquirir una vivienda inflada de precio o invertir en títulos basura desconociendo la calidad de la inversión (Feldkircher, 2014).

La educación financiera es definida como el proceso mediante el cual los usuarios comprenden mejor los productos y riesgos financieros, desarrollando habilidades para tomar decisiones mejor informadas con los riesgos que estas decisiones acarrean (OECD, 2005). Dada su importancia, debe ser impartida a los ciudadanos de cualquier edad ya que se trata de un proceso continuo a lo largo de toda la vida, donde se recomienda empezar desde una temprana edad (Domínguez, 2013).

La mayoría de países desarrollados han implementado diversas estrategias donde, pese a los altos niveles de ingreso y estándares educativos, es clara la deficiente educación financiera de las personas, evidenciada por las diferentes crisis económicas. Es así como la Comisión Europea en un informe para el mercado común anunciaba la educación financiera como componente esencial de su labor para asegurar que el mercado único pueda aportar beneficios directos a sus ciudadanos, especialmente capacitándolos no sólo para buscar los mejores servicios financieros, sino también para comprender algunos conceptos básicos esenciales de las finanzas personales (Comisión Europea, 2007). También se tienen medidas en países emergentes, uno de ellos es Colombia, donde el Ministerio de Educación apoyado por la asociación bancaria del mismo país, desarrollaron un plan para promover la educación financiera desde la escuela con el propósito de desarrollar en los niños, niñas, adolescentes y jóvenes los conocimientos, las habilidades y las actitudes necesarias para la toma de decisiones financieras cotidianas (Mineducación, 2014). En concordancia con la regulación vigente en el país, cada establecimiento educativo deberá definir la forma de articular la educación financiera a su proyecto educativo institucional.

A pesar de estos esfuerzos, las estadísticas siguen mostrando una historia diferente. Mientras el $90 \%$ de los estudiantes quieren aprender finanzas, el $80 \%$ de los profesores no se siente apto para enseñar esta asignatura (Kiyosaki, 2015). Kiyosaki reporta la existencia de una crisis educativa por la ausencia de educación financiera en las escuelas, lo cual fue corroborado por grandes economistas, incluyendo el expresidente de la Reserva Federal de Estados Unidos, Ben Bernanke, quien también enunció a la ignorancia financiera como la principal causante de la crisis financiera del año 2008.

La educación como proceso social en permanente innovación, debe adaptarse continuamente a los requerimientos de la dinámica de la sociedad moderna y de la misma forma debe incorporar otros aspectos importantes como medioambiente, cambio climático y huella de carbono (Valderrama et al., 2011); la educación financiera constituye un tema transversal que también debe introducirse desde la escuela primaria hasta ser dominada con propiedad por profesionales de la ingeniería y otras áreas.

Se requiere por tanto un cambio de actitud donde la educación financiera sea transversal en las diferentes áreas del conocimiento de la ingeniería. Cada curso debe tener un capítulo donde se analicen los aspectos económicos y financieros, pues el mundo actual no sólo requiere un adecuado diseño y fabricación de equipos, si no que esto se logre a un costo óptimo. De la misma forma se debe entender el entorno macroeconómico y el impacto de variables importantes como la inflación, la devaluación y las tasas de interés a nivel personal y en el sector empresarial. Sin embargo, este ideal es muy difícil de conseguir en el entorno actual del sistema educativo, especialmente de los países emergentes. 
Dada la dificultad de abordar el tema de forma transversal, en este artículo se propone que el curso de ingeniería económica (o cursos equivalentes) se oriente desde el comienzo a la educación financiera, mediante la aplicación de todos los conceptos a las finanzas personales. Se muestran los aspectos más relevantes de la educación financiera y conceptos generales de la ingeniería económica, introduciendo la importancia de los proyectos de aula o trabajos guiados de semestre como una forma de aplicar estos conceptos a casos personales de cada estudiante. Desde el punto de vista de los autores, esto permitirá interiorizar cada uno de los conceptos, lo que además de contribuir a su educación financiera, le permitirá a la vez aplicarlos fácilmente a otras áreas de la ingeniería.

\section{IMPORTANCIA Y EFECTOS DE LA EDUCACIÓN FINANCIERA}

La educación financiera entrega herramientas a las personas para tomar decisiones efectivas que permitan mejorar su bienestar económico. Su importancia es cada vez mayor dado el número creciente y complejo de productos que ofrecen los mercados financieros, con el fin de atender las necesidades de rentabilidad de una población cada vez más preocupada por los cambios en los sistemas de pensiones que les impone el reto del retiro a mayor edad con unos menores ingresos. La amplia gama de productos que ofrecen mayor rentabilidad han hecho caer a los más incautos y consecuencia de ello ha sido la última burbuja hipotecaria y del mercado de valores con los resultados conocidos especialmente en la población más vulnerable. La educación financiera parte de una adecuada planeación financiera que le permita a las personas elaborar el plan de acción para cumplir sus objetivos personales tanto en el corto, como mediano y largo plazo. El plan va a determinar qué hacer, cómo hacerlo, cuánto tiempo tardará y la viabilidad real que tiene para poder conseguir lo que quiere (Samper et al., 2010).

La educación financiera comprende tres aspectos clave: i) Adquirir un conocimiento adecuado en materia de finanzas; ii) Desarrollar las competencias que permitan utilizar los conocimientos en beneficio propio; y iii) Ejercer la responsabilidad financiera mediante una gestión adecuada de las finanzas personales. De esta forma, la importancia de la educación financiera no se limita a las personas mayores, sino que se debe empezar a tomar conciencia de la misma desde la niñez haciendo énfasis en cada una de las fases del ciclo escolar hasta llegar a la universidad. En este contexto, desde la década de 1990 se han desarrollado diferentes estudios sobre la educación financiera y sus efectos. Los principales estudios y sus resultados se resumen en la Tabla 1.

Los estudios referidos en la Tabla 1 evidencian la necesidad de preparar a nuestros estudiantes para situaciones del mundo real, la cual fue la razón principal de incluir la educación financiera en las pruebas PISA (OECD, 2012). En los últimos años se ha hecho más evidente la importancia de la misma tanto en las economías emergentes como desarrolladas debido a la mayor longevidad de la población y unos mercados financieros, cada vez más especializados con un mayor número de productos financieros, que atraen a los ciudadanos con expectativas de grandes rentabilidades. En consecuencia, la educación financiera se ha convertido en un elemento importante de estabilidad económica y desarrollo en todos los países. En la tabla 2 se ilustran algunos ejemplos de países que han adoptado la educación financiera en sus centros de enseñanza (Banco de España, 2013). Algunos aspectos que demuestran la necesidad de una educación financiera sólida para todos los individuos son los siguientes:

\section{La Cultura del Ahorro y la Inversión}

El punto de partida de la educación financiera es la cultura del ahorro y la inversión, a través de la misma se podrá realizar una adecuada planeación que permita conseguir las metas fijadas en el futuro. El éxito de estas metas requiere que independiente del nivel de ingresos, cada individuo adopte la costumbre de ahorrar como mínimo entre el $10 \%$ y $20 \%$ de sus ingresos brutos (Samper et al., 2010). Esto se logra con un ejercicio de análisis de ingresos y egresos, donde se dé prioridad a los gastos más importantes de tal forma que quede una cantidad suficiente para ahorrar dentro del rango recomendado.

Luego se hace necesario invertir adecuadamente esos ahorros de tal forma que se pueda obtener la mejor rentabilidad con un nivel de riesgo adecuado. Se requiere por lo tanto del conocimiento de los mercados financieros con productos que van desde una cuenta de ahorros, certificados de depósito a término, pasando por inversiones en bolsa de valores hasta productos más exóticos que se encuentran en el mercado de derivados. Entre más sofisticado y complejo sea el producto se puede lograr una mejor rentabilidad pero acompañada también de un riesgo mayor.

\section{La Necesidad de Mejorar los Ingresos a la Edad de Retiro}

Cada vez se escuchan más noticias sobre las crisis de los sistemas de pensiones en gran parte de los países tanto emergentes como desarrollados. El déficit pensional en los sistemas públicos tiene un peso notable en los presupuestos de los estados incrementando el endeudamiento y afectando otras inversiones 
sociales. Como resultado se está transfiriendo la responsabilidad de la pensión por vejez a los fondos privados donde el mismo ciudadano es responsable de la edad de retiro y los ingresos a recibir cuando ello ocurra. Algo similar ocurre con los servicios de salud, cada vez más deficientes y costosos que obliga a las personas a adquirir programas y seguros complementarios.

Tabla 1: Principales estudios sobre educación financiera y sus efectos

\begin{tabular}{|c|c|c|}
\hline Referencia & Tipo de estudio & Principales resultados y observaciones \\
\hline $\begin{array}{l}\text { (Bernheim et al, } \\
\text { 1997) }\end{array}$ & $\begin{array}{l}\text { Efectos a largo plazo de la } \\
\text { educación financiera en las } \\
\text { escuelas }\end{array}$ & $\begin{array}{l}\text { La tasa de ahorro y riqueza de individuos que asistieron a escuelas } \\
\text { donde se implementaron programas de educación financiera es } \\
\text { significativamente mayor a la de los individuos que no participaron de } \\
\text { estos programas. }\end{array}$ \\
\hline $\begin{array}{l}\text { (Bernheim y } \\
\text { Garret, 2003) }\end{array}$ & $\begin{array}{l}\text { Efecto de la educación } \\
\text { financiera en el lugar de } \\
\text { trabajo. }\end{array}$ & $\begin{array}{l}\text { Las tasas de ahorro aumentan en relación a la educación financiera } \\
\text { de los encuestados. La probabilidad de participar en programas de } \\
\text { ahorro para complementar la mesada de jubilación aumenta en } \\
\text { empleados con educación financiera. }\end{array}$ \\
\hline $\begin{array}{l}\text { (Lusardi y } \\
\text { Mitchell, 2006) }\end{array}$ & $\begin{array}{l}\text { Efecto de educación } \\
\text { financiera en individuos } \\
\text { mayores de } 50 \text { años }\end{array}$ & $\begin{array}{l}\text { Los conocimientos a nivel financiero en la población bajo estudio son } \\
\text { escasos. Existe una correlación positiva entre conocimientos } \\
\text { financieros y planificación de la jubilación. }\end{array}$ \\
\hline $\begin{array}{l}\text { (Lusardi y } \\
\text { Mitchell, 2007) }\end{array}$ & $\begin{array}{l}\text { Analiza la acumulación de } \\
\text { riqueza de individuos } \\
\text { nacidos entre } 1950 \text { y } 1960\end{array}$ & $\begin{array}{l}\text { Después de controlar factores sociodemográficos encuentran que la } \\
\text { planificación para la jubilación está directamente relacionada con los } \\
\text { conocimientos financieros y niveles de riqueza. A mayor planificación } \\
\text { existe más inversión en instrumentos financieros sofisticados y mayor } \\
\text { riqueza. }\end{array}$ \\
\hline $\begin{array}{l}\text { (Bucher- } \\
\text { Koenen y } \\
\text { Lusardi 2011) }\end{array}$ & $\begin{array}{l}\text { Realiza encuesta en } \\
\text { hogares alemanes sobre } \\
\text { conocimientos financieros }\end{array}$ & $\begin{array}{l}\text { Los conocimientos financieros de los hogares alemanes son } \\
\text { moderados, siendo mayores en Alemania del Oeste. Se encuentra } \\
\text { nuevamente una correlación positiva entre los conocimientos } \\
\text { financieros y la planificación para la jubilación. }\end{array}$ \\
\hline $\begin{array}{l}\text { (Hastings y } \\
\text { Tejeda-Ashton, } \\
\text { 2008) }\end{array}$ & $\begin{array}{l}\text { Relación entre educación } \\
\text { financiera e inversión en } \\
\text { México }\end{array}$ & $\begin{array}{l}\text { En el contexto de inversión en fondos de seguridad social los } \\
\text { encuestados con mayores conocimientos financieros le dan mayor } \\
\text { importancia a las comisiones que aquellos con menor conocimiento } \\
\text { financiero. Estos últimos encuestados son más sensibles a los } \\
\text { cambios de formato que a información relevante con respecto a su } \\
\text { inversión. }\end{array}$ \\
\hline $\begin{array}{l}\text { (Stango y } \\
\text { Zinman, 2009) }\end{array}$ & $\begin{array}{l}\text { Educación financiera y } \\
\text { nivel de endeudamiento de } \\
\text { individuos y hogares }\end{array}$ & $\begin{array}{l}\text { Los consumidores con baja educación financiera no interpretan } \\
\text { adecuadamente el efecto de las tasas exponenciales, lo que resulta } \\
\text { en que subestimen valores futuros de inversiones o préstamos. } \\
\text { Individuos con baja educación financiera tienden a adquirir préstamos } \\
\text { con más altas tasas de interés y costos de comisiones mayores. }\end{array}$ \\
\hline $\begin{array}{l}\text { (Hilgert et al, } \\
\text { 2003) }\end{array}$ & $\begin{array}{l}\text { Educación financiera en } \\
\text { los hogares }\end{array}$ & $\begin{array}{l}\text { La adecuada gestión de deudas y crédito se encuentra relacionada } \\
\text { con el nivel de educación financiera del individuo. Individuos con } \\
\text { mayor educación financiera tienen menores niveles de deudas. }\end{array}$ \\
\hline $\begin{array}{l}\text { (Van Rooij et al, } \\
2007)\end{array}$ & $\begin{array}{l}\text { Conexión de los niveles de } \\
\text { educación financiera con la } \\
\text { inversión en mercados de } \\
\text { valores en hogares } \\
\text { holandeses }\end{array}$ & $\begin{array}{l}\text { La educación financiera difiere sustancialmente en cuanto a la edad, } \\
\text { género y profesión de los encuestados. Se sugiere que los programas } \\
\text { de educación financiera deben ser orientados a grupos específicos de } \\
\text { la población. La falta de conocimientos financieros disminuye la } \\
\text { probabilidad de participación en los mercados financieros. }\end{array}$ \\
\hline $\begin{array}{l}\text { (Van Rooij et al, } \\
\text { 2011) }\end{array}$ & $\begin{array}{l}\text { Estudio sobre educación } \\
\text { financiera y acumulación } \\
\text { de riqueza }\end{array}$ & $\begin{array}{l}\text { Muestra que hay correlación positiva entre la acumulación de riqueza } \\
\text { y la educación financiera en los hogares. Las dos razones básicas } \\
\text { son el hecho de que los individuos mejor educados financieramente } \\
\text { son proclives a invertir en renta variable y tienen mayor propensión a } \\
\text { planificar para la jubilación. }\end{array}$ \\
\hline $\begin{array}{l}\text { (Drexler et al, } \\
\text { 2014) }\end{array}$ & $\begin{array}{l}\text { Impacto de la formación } \\
\text { financiera en pequeños } \\
\text { negocios de República } \\
\text { Dominicana }\end{array}$ & $\begin{array}{l}\text { Mayores conocimientos contables y financieros tienen un efecto } \\
\text { positivo en los pequeños negocios. En este tipo de negocios la } \\
\text { educación financiera basada en reglas simples y generales resulta } \\
\text { tener mayor impacto que el manejo de reglas sofisticadas. }\end{array}$ \\
\hline
\end{tabular}

Un análisis de las principales características de los sistemas de pensiones basados en cuentas de ahorro individual es realizado por Mamani y Garay (2010). Se hace énfasis en el modelo chileno, pionero en Latinoamérica y que ha sido el referente para las reformas en otros países como México, Perú y Colombia. Se resalta como el sistema chileno ha traído más ventajas que desventajas para el estado, debido a que parte de esos recursos se utilizan para la construcción de obras públicas y han sido un apoyo importante para la reactivación del mercado de capitales. Sin embargo, se critica la falta de solidaridad del sistema que hacen necesarias reformas al mismo. 
Tabla 2: Países donde se imparte educación financiera en las escuelas

\begin{tabular}{|c|l|}
\hline País & \multicolumn{1}{c|}{ Descripción } \\
\hline Nueva Zelanda & $\begin{array}{l}\text { A partir de } 2009 \text { estudiantes de Lengua, Ciencias Sociales, Matemáticas y Tecnología con } \\
\text { edades entre } 5 \text { y } 14 \text { años participan de un plan de estudios que se imparte de forma transversal } \\
\text { y pretende dar capacitación financiera en las escuelas. }\end{array}$ \\
\hline Australia & $\begin{array}{l}\text { Desde } 2005 \text { se imparte educación financiera, en las escuelas de manera obligatoria, en } \\
\text { asignaturas de Matemáticas, Ciencias, Humanidades y Tecnología para niños de } 3 \text { a } 10 \text { años. } \\
\text { En } 2013 \text { se llevó a cabo la reforma del currículum escolar donde se integra la capacitación } \\
\text { financiera para alumnos entre } 10 \text { y } 12 \text { años. }\end{array}$ \\
\hline Escocia & $\begin{array}{l}\text { Desde } 2008 \text { la educación financiera es obligatoria en el currículum de las escuelas. No se } \\
\text { incluye en materas específicas y se aplica en primaria y secundaria. }\end{array}$ \\
\hline Inglaterra & $\begin{array}{l}\text { A partir de } 2014 \text { la educación financiera se hace obligatoria en las escuelas. Antes de esta fecha } \\
\text { la capacitación financiera solo era parte de algunos contenidos en Matemáticas y Ciudadanía. }\end{array}$ \\
\hline Brasil & $\begin{array}{l}\text { En } 2010 \text { se realiza programa piloto de capacitación financiera en colegios públicos. Abarca más } \\
\text { de } 30000 \text { estudiantes en } 900 \text { colegios. }\end{array}$ \\
\hline España & $\begin{array}{l}\text { Desde } 2010 \text { se desarrollan programas piloto para incluir educación financiera en la secundaria. } \\
\text { En } 2013 \text { se proponen estrategias nacionales de educación financiera que incluye la enseñanza } \\
\text { de la misma en escuelas. }\end{array}$ \\
\hline Colombia & $\begin{array}{l}\text { En } 2014 \text { el Ministerio de Educación con apoyo de la asociación bancaria, desarrolla un plan para } \\
\text { promover la educación financiera desde la escuela. }\end{array}$ \\
\hline
\end{tabular}

Estas reformas se hacen cada vez más necesarias dado que el aumento de las expectativas de vida de la población se ha reflejado en unos menores ingresos para quienes han llegado a la edad de retiro. Estos ingresos, que en muchos casos son inferiores al 50\% del último salario devengado, obligan a que cada persona desde el comienzo de su vida laboral, empiece a planificar como va a compensar esta drástica disminución en los ingresos a la edad de retiro.

\section{La Educación de los Hijos}

El déficit fiscal existente especialmente en los países emergentes, ocasionado en gran parte por problemas de endeudamiento y carga pensional; ha sido el causante de la afectación de otras inversiones prioritarias como salud y educación. No se puede desconocer el papel de la educación pública en aliviar el alto grado de inequidad existente en las economías emergentes, pues es a través de la misma que las personas de menores ingresos pueden aspirar a mejorar su calidad de vida.

La conciencia de la importancia de la educación de los hijos, acompañada del incremento en la expectativa de vida y mayores gastos sociales; obliga a los individuos ahorrar para el futuro y a buscar las mejores rentabilidades. Siempre se ha dicho que la educación es la mejor de las inversiones; sin embargo, algunas experiencias en los países desarrollados han demostrado que esta premisa no siempre se cumple, especialmente cuando se adquiere una deuda muy alta para pagar una universidad costosa y en una carrera que no va a brindar los ingresos suficientes. La mejor decisión para los padres es planear la educación de sus hijos, ojalá desde muy temprana edad, de tal forma que no tengan problemas financieros al momento en que ellos lleguen a su vida universitaria.

\section{La Planeación Tributaria}

Los impuestos constituyen la forma de financiar el presupuesto de gastos e inversión de cada estado, por tal razón corresponde a cada ciudadano el deber de tributar. Estos tributos dependen de la legislación vigente en cada país lo que hace indispensable conocer los decretos que correspondan o buscar la asesoría de expertos legales y contables, especialmente en aquellos aspectos referentes a inversiones o gastos personales que son deducibles del impuesto a la renta.

En general existe un alto nivel de tributación en la mayoría de los países, el cual tiene tendencia a aumentar por las reformas fiscales permanentes que buscan cubrir el déficit producido por la mayor demanda de gastos sociales y de servicio de la deuda. Por otra parte, el estatuto tributario de cada país establece también una serie de incentivos o deducciones fiscales que buscan beneficiar a determinados sectores económicos. Esto obliga a realizar una adecuada planeación de los impuestos que permita aprovechar los descuentos vigentes y así pagar lo mínimo posible dentro del marco legal. 


\section{Otras Metas Personales}

Todas las personas tienen sueños o metas por cumplir. Adquirir vivienda propia, un viaje a otro continente o un emprendimiento personal son algunos ejemplos. Lo más recomendable es elaborar un plan de ahorro e inversión para disponer de los recursos necesarios, especialmente cuando se trata de lujos o viajes de placer; sin embargo, algunos sueños pueden ser oportunidades de inversión que requerirán complementarse con una deuda. En cualquier caso la deuda debe ser manejable, es decir, la cuota del préstamo debe ser cubierta con los ingresos provenientes del emprendimiento o ahorros obtenidos por arrendamiento (en caso de préstamo hipotecario).

La importancia de la educación financiera se hace cada vez más evidente por esta cadena de necesidades de ahorro de los individuos con mayor demanda de productos financieros. Se requiere de unos ciudadanos mejor informados, que conozcan la diversidad de productos, el funcionamiento de cada uno, los mecanismos de rentabilidad y ante todo los riesgos inherentes. Todos estos factores también impactan directamente el desarrollo de los países, pues unas adecuadas decisiones financieras de los individuos, conllevan un sector financiero más eficiente que requiera menores costos del estado en regulación y supervisión.

\section{LA INGENIERÍA ECONÓMICA Y LA EDUCACIÓN FINANCIERA}

La ingeniería económica se puede definir como el conjunto de técnicas matemáticas para tomar decisiones económicas en los ámbitos industrial, comercial y de servicios. Estas técnicas deben ser del conocimiento de todos los profesionales de cualquier rama de la administración y la ingeniería, ya que diariamente se enfrentan en su trabajo a situaciones en las cuales tienen que tomar decisiones que involucran dinero.

Su desarrollo fue paralelo al rápido crecimiento industrial de la segunda mitad del siglo XX, donde se creó la necesidad de contar con técnicas de análisis económico orientadas a la toma de decisiones en las empresas. Es así como se empezaron a utilizar conceptos como valor del dinero en el tiempo, tasa de interés, capitalización, pagos anuales, amortizaciones, etc., en el ámbito industrial y particularmente en el área productiva de las empresas, con los cuales se generó una disciplina que recibió el nombre de ingeniería económica (Vidaurri, 2013).

Estos conceptos de la ingeniería económica se hacen fundamentales para la planeación financiera, punto de partida de la educación financiera, la cual requiere de la toma de decisiones adecuadas desde el corto hasta el largo plazo. Según Samper et al. (2010), se requieren los siguientes cinco pilares básicos para lograr el éxito en la planeación financiera: i) El tiempo; ii) Interés compuesto; iii) Conciencia de la inflación; iv) Optimización tributaria; v) Manejo del endeudamiento. Estos pilares clave para tomar decisiones efectivas necesitan del dominio de las técnicas de la ingeniería económica.

De forma rutinaria se debe enfrentar el desafío de tomar decisiones financieras Este reto va desde una persona que devenga un salario por pequeño que sea, hasta el propietario de una gran empresa (Blank y Tarquin, 2012). Es así como los conceptos de la ingeniería económica, además de su aplicación en las empresas, también son útiles en la vida cotidiana de las personas, por ello resulta fundamental su comprensión para la toma de decisiones que tendrán impacto directo en la solidez financiera de las empresas y los individuos. A continuación se presentan de forma introductoria algunos conceptos básicos de la ingeniería económica de gran utilidad en las finanzas personales (Blank y Tarquin, 2012; Meza. 2013; Vidaurri (2013):

\section{La tasa de interés y el valor del dinero en el tiempo}

El interés es una especie de canon de arrendamiento que se cobra por el dinero y por lo tanto la tasa de interés representa la rentabilidad o valorización del dinero con el tiempo. Estos dos conceptos van de la mano y se constituyen como unos de los más importantes de la ingeniería económica. Esta relación se expresa en (1) donde el valor futuro equivalente $(F)$ a una cantidad monetaria de hoy $(P)$ con una tasa de interés por periodo (I) para $n$ periodos, se calcula como:

$$
F=P(1+i)^{n}
$$

\section{Las series uniformes}

Corresponden a un conjunto de pagos iguales hechos a intervalos uniformes de tiempo. Las amortizaciones periódicas son de gran utilidad cuando se requiere determinar la cuota a pagar por un préstamo porque constituyen el sistema de amortización más común en los créditos comerciales y bancarios. En (2) se muestra la forma de calcular dichas cuotas, donde $A$ es el monto de la cuota a pagar a partir del primer periodo y $P$ es 
el valor actual del préstamo.

$$
A=P \frac{i(1+i)^{n}}{(1+i)^{n}-1}
$$

Otra variante corresponde al cálculo de los ahorros periódicos constantes que se deben realizar cuando se pretende obtener una cantidad de dinero en el futuro que permita cumplir una de las metas de la planeación financiera. En este caso se utiliza la ecuación (3), donde $A$ es el ahorro constante entre los periodos 1 y n, $F$ es la cantidad requerida en el futuro.

$$
A=F \frac{i}{(1+i)^{n}-1}
$$

\section{Evaluación de Alternativas de Inversión}

Una inversión es la asignación de recursos en el presente con el objetivo de obtener unos beneficios futuros, por lo cual la decisión de invertir debe ser cuidadosamente analizada, especialmente cuando se dispone de diferentes alternativas en el mercado. En la ingeniería económica se han desarrollado técnicas que permiten comparar alternativas de inversión y de esta forma poder seleccionar la más conveniente. Las más utilizadas son el valor presente neto y la tasa interna de rentabilidad (Meza, 2013; Vidaurri; 2013).

El valor presente neto es el resultado de comparar todos los flujos de caja de la inversión en dinero de hoy, por lo cual, un valor presente neto positivo significa que la inversión es atractiva ya que los ingresos obtenidos son superiores al monto invertido. Este indicador se calcula usando la ecuación (4), donde VPN es el valor presente neto; $F C_{j}$ es el flujo de caja para el periodo j; $i$ es la tasa de interés mínima aceptable de rentabilidad o en otras palabras, el costo de los recursos que se emplean en la inversión.

$$
V P N=\sum_{j=0}^{j=n} \frac{F C_{j}}{(1+i)^{j}}
$$

La tasa interna de rentabilidad corresponde a la tasa de interés que rinden los dineros que permanecen invertidos. Este valor se obtiene a partir de (4) y corresponde a la tasa de interés $i$ que iguala el valor presente neto a cero.

\section{El Manejo del Riesgo}

La ingeniería económica toma decisiones en el presente basándose en estimaciones de sucesos futuros, lo cual tiene de forma inherente un error por la dificultad de predecirlo. Por lo tanto, cualquier análisis económico debe involucrar el riesgo teniendo en cuenta que los resultados finales pueden ser diferentes a los esperados. Desde el punto de vista de las finanzas personales, el riesgo se puede definir como la posibilidad de que un pago futuro no se dé como está previsto y hay que tener en cuenta que los mercados premian el riesgo con mayor rentabilidad; de ahí que a pesar que siempre se busquen buenas rentabilidades, se debe desconfiar cuando éstas son muy altas ya que el riesgo puede ser hasta de perder la totalidad del capital invertido.

\section{PROYECTOS QUE PUEDEN DESARROLLAR LOS ALUMNOS}

El principal objetivo de la educación financiera es entender lo que sucede en nuestro entorno económico, la forma en que nos afecta y como se pueden aprovechar las noticias diarias para el beneficio propio y logro de proyectos personales. Una forma apropiada de asimilar los conceptos de educación financiera puede ser mediante proyectos de aula o trabajos guiados de semestre (Ballesteros y Moral, 2014; Valderrama y Valderrama, 2014; Torres et al., 2016). Tal como lo señalan los autores, estas metodologías buscan aprovechar los conocimientos recopilados en los cursos, a través de las cuales el estudiante va adquiriendo la capacidad de relacionar los conceptos teóricos con la experiencia práctica para solucionar problemas reales.

Una experiencia relacionada con el trabajo en proyectos la tuvo uno de los autores en el curso mercados y bolsa de energía, en el cual los estudiantes divididos por grupos eligen un simulador con una cuenta de negociación virtual. Esta cuenta virtual trabaja paralela al mercado real, de tal forma que permite aplicar todos los conocimientos adquiridos en el curso determinando el portafolio adecuado y aplicando las metodologías de análisis fundamental y análisis técnico con el fin de seleccionar los activos a invertir y el momento adecuado para hacerlo. Los resultados de esta experiencia se midieron con la evaluación que los 
estudiantes realizaron del curso. En la Tabla 3 se muestra dicha evaluación con el promedio de los resultados correspondientes a siete semestres que lleva implementándose la metodología, la cual se compara con el promedio de los siete semestres anteriores al periodo de implementación (la calificación se realiza en escala de cero a cinco). La evaluación muestra una mejoría notable en la percepción de los estudiantes en cuanto a la capacidad de despertar interés, el empleo de recursos didácticos y el apoyo a las actividades de aprendizaje independientes. Este último ítem es una indicación de que esta metodología requiere de una mayor dedicación por parte del profesor.

Tabla 3: Evaluación de los estudiantes a un curso de ingeniería (efecto de incorporar proyectos de aula)

\begin{tabular}{|c|c|c|}
\hline Evaluación Del Curso & Antes & Después \\
\hline La metodología del curso tiene la capacidad de despertar el interés de los estudiantes & 4.14 & 4.59 \\
\hline Se emplean recursos didácticos adecuados & 4.15 & 4.62 \\
\hline El profesor es eficiente en el uso del tiempo de clase y actividades & 4.45 & 4.65 \\
\hline Existe orden, coherencia y claridad en la exposición de los temas & 4.23 & 4.59 \\
\hline El profesor es puntual y asiste a todas las sesiones de clase & 4.66 & 4.61 \\
\hline Se apoyan las actividades de aprendizaje independientes & 4.20 & 4.60 \\
\hline Es importante el curso dentro de su plan de estudios & 4.62 & 4.69 \\
\hline El curso es interesante y actualizado en sus contenidos & 4.48 & 4.74 \\
\hline Considera usted que se lograron los objetivos formulados en el curso o actividad curricular & 4.41 & 4.66 \\
\hline
\end{tabular}

El aprendizaje por proyectos constituye de esta forma una estrategia integral de formación contribuyendo al desarrollo de destrezas en los individuos. Para incorporar la educación financiera, se propone que los estudiantes elijan proyectos a largo plazo que representen una meta importante en su vida. Algunos ejemplos son mejorar los ingresos del retiro, lograr una jubilación a más temprana edad, la educación de los hijos, un viaje a otro continente o un emprendimiento empresarial. Ello obliga a que cada estudiante establezca la planificación financiera que le permita alcanzar ese objetivo. Un ejemplo de modelo de planeación financiera a seguir es el propuesto por Samper et al. (2010), el cual comprende los siguientes pasos: (i) Diagnóstico de la situación financiera actual, (ii) Identificación clara de lo que quiere lograr, (iii) Implementación de un plan de acción, (iv) Verificación de su progreso.

El modelo de planeación financiera a seguir obliga a que el estudiante se apropie de conceptos claves de la ingeniería económica como es el valor del dinero en el tiempo, la tasa de interés de oportunidad, la inflación, la devaluación, el endeudamiento, la optimización tributaria, análisis de riesgo y los principales métodos de evaluación financiera de proyectos.

Cada estudiante debe implementar un plan de acción partiendo de la meta fijada y el horizonte de tiempo para lograrla. Luego, elabora la evaluación financiera o calcula la cantidad de dinero requerida para conseguir la meta mediante el uso de los conceptos de ingeniería económica. Finalmente, establece el monto de ahorro y las acciones necesarias para iniciar el proceso de forma paralela al desarrollo del curso.

Se propone partir del flujo de presupuesto mensual de ingresos y egresos e identificar cuanto se puede ahorrar. Luego debe investigar las diferentes alternativas de inversión a corto, mediano y largo plazo con las respectivas rentabilidades y riesgos asociados. De esta forma se encontrará la mejor forma de invertir sus ahorros y alcanzar su meta aplicando los conceptos de ingeniería económica y logrando una educación financiera adecuada.

\section{CONCLUSIONES}

Las finanzas personales cobran cada vez mayor importancia en el mundo moderno dado que los estados están descargando en sus ciudadanos la responsabilidad de aspectos tan importantes como la pensión y gastos de salud y educación, entre otros. De forma simultánea, los mercados financieros ofrecen un número creciente de productos altamente sofisticados que obligan a los individuos a estudiarlos y comprender su rentabilidad y riesgos inherentes.

A pesar de las iniciativas de cada país por una adecuada educación financiera, las crisis económicas recientes evidencian su deficiente divulgación desde la escuela hasta la misma universidad. Los ingenieros no son ajenos a este fenómeno debido a la necesidad de una formación financiera no sólo para su vida personal sino también para el ejercicio profesional. Por esta razón, los conceptos del curso de ingeniería económica se pueden utilizar de forma simultánea para la educación financiera de los estudiantes de ingeniería mediante el desarrollo de proyectos de aula o trabajos guiados de semestre. 
No se puede desconocer el papel fundamental del docente en esta función. Se hace necesario que tome mayor conciencia de la importancia de la educación financiera y enriquezca sus cursos con casos prácticos y proyectos con los estudiantes. Estas actividades requieren del concurso del estado e instituciones responsables mediante la capacitación, promoción y estimulación de la labor del profesorado en los diferentes campos de esta formación.

\section{AGRADECIMIENTOS}

Los autores agradecen el apoyo financiero recibido de la Universidad de Antioquia por medio del proyecto "Sostenibilidad 2016-2017".

\section{REFERENCIAS}

Banco de España, Comisión Nacional de Mercado de Valores. Plan de educación financiera 2013-2017, http://www.cnmv.es/DocPortal/Publicaciones/PlanEducacion/PlanEducacion13_17.pdf. Acceso: 11 Febrero (2016)

Bartram S.M. y G.M. Bodnar, No place to hide: the global crisis in equity markets in $2008 / 2009$, Journal of International Money and Finance, Elsevier, 28 (8), 1246-1292 (2009)

Balleresteros, M. y A. Moral, Using simulation software to implement an active learning methodology in the university teaching, International Journal of Innovation in Education, 1 (1), 87-98 (2014)

Bernheim, D. y D. Garret, The effects of financial education in the workplace: evidence form a survey of households, Journal of Public Economics, Elsevier, 87(1), 1487-1519 (2003)

Bernheim D., D. Garret, and D. Maki, Education and saving: the long-term effects of high school financial curriculum mandates, Journal of Public Economics, Elsevier, 80(3), 435-465 (1997)

Blank, L. y A. Tarquin, Ingeniería económica, 7ª̣ edición, 3-9, McGraw-Hill, México (2012)

Bucher-Koenen T. y A. Lusardi, Financial literacy and retirement planning in Germany. National Bureau of Economic Research (NBER) Working Paper Nº 17110 (2011)

Comisión Europea, Comunicación de la comisión: La educación financiera. COM, Bruselas, Bélgica (2007). Descargado: 24 de Julio (2016)

Domínguez, J. M., Educación financiera para jóvenes: Una visión introductoria, Serie Documentos de Trabajo. Universidad de Alcalá, España (2013). Descargado 2 de Junio (2016)

Drexler A., G. Fischer, y A. Schoar., Keeping it simple: Financial Literacy and Rules of Thumb. American Economic Journal, Applied Economics, 6 (2), 1-31 (2014)

Dwyer G.P y J.R. Lothian, International and historical dimensions of the financial crisis of 2007 and 2008, Journal of International Money and Finance, Elsevier, 31 (1), 1-9 (2012)

Feldkircher, M., The determinants of vulnerability to the global financial crisis 2008 to 2009: credit growth and other sources of risk. Journal of International Money and Finance, Elsevier, 43 (1), 19-49 (2014)

Hastings J. y L. Tejeda-Ashton, Financial literacy, information and demand elasticity: survey and experimental evidence from Mexico, NBER Working, Paper № 14538 (2008)

Hilgert, M., J. Hogarth, y S. Beverly, Household Financial Management: The Connection Between Knowledge and Behaviour, Federal Reserve Bulletin, 309-322 (2003)

Kiyosaki, R., Despierta el genio financiero de tus hijos, 1a edición, 97-130, Penguin Random House, Barcelona, España (2015)

Lusardi A. y O. Mitchell, Financial literacy and planning: implications for retirement wellbeing. National Bureau of Economic Research (NBER) Working Paper Nº 2005-108 (2006)

Lusardi A. y O. Mitchell, Baby boomer retirement security: the role of planning, financial literacy and housing wealth, Journal of Monetary Economics, 54 (1), 205-224 (2007)

Mamani, A., H. y S. Garay, las implicancias de los actuales sistemas previsionales basados en cuentas de capitalización individual, análisis del caso chileno, Estudios Sociales, 18 (36), 78-98 (2010) 
Melvin M. y M.P. Taylor, The global financial crisis: causes, threats and opportunities, introduction and overview, Journal of International Money and Finance, Elsevier, 28 (8), 1243-1245 (2009)

Meza, J. J., Evaluación financiera de proyectos, 3ª edición, 133-161, ECOE Ediciones, Bogotá (2013)

MINEDUCACIÓN, Mi plan de vida y mi futuro. Orientaciones pedagógicas para la educación económica y financiera. Ministerio de Educación de Colombia, Bogotá (2014). Descargado: 8 de Agosto (2016)

OECD, Improving financial literacy: Analysis of issues and policies, OECD Publishing, París, Francia (2005). Acceso: 15 de Julio (2016)

OECD, PISA 2012, Assessment and analytical framework, OECD Publishing, París, Francia (2012). Acceso: 20 de Julio (2016)

Samper, M. J., C. Pinto, J. Arenas y M. Roca, Mis finanzas personales, 1a edición, 37-71, Aguilar, Bogotá, Colombia (2010)

Stango, V. y J. Zinman, Exponential Growth Bias and Household Finance, Journal of Finance, 64 (6), $2807-$ 2849 (2009)

Torres, J., D., D. Acevedo y P. M. Montero, Proyectos de aula semestrales como estrategia pedagógica para la formación en ingeniería, Formación Universitaria, 9 (3), 23-30 (2016)

Valderrrama, J., O., C. Espíndola y R. Quezada, Huella de carbono, un concepto que no puede estar ausente en cursos de ingeniería y ciencias, Formación Universitaria, 4 (3), 3-12 (2011)

Valderrrama, A. y J. Valderrama, Trabajo semestral guiado: un buen complemento al estudio y aprendizaje en cursos de ciencia y de ingeniería, Formación Universitaria, 7 (3), 3-10 (2014)

Van Rooij, M., A. Lusardi, y R. Alessie, Financial Literacy and Stock Market Participation. National Bureau of Economic Research (NBER) Working Paper N 13-565 (2007)

Van Rooij, M., Lusardi, A. y Alessie, R. Financial Literacy, Retirement Planning, and Household Wealth, National Bureau of Economic Research (NBER) Working Paper N 17-339, (2011)

Vidaurri, H. M., Ingeniería económica básica, 1ª edición, 1-6. Cengage Learning, México (2013) 